Герштейн Е.С. • Иванников А.А. ${ }^{2}$ Чанг В.Л. ${ }^{2}$ • Огнерубов Н.А. ${ }^{2}$ Давыдов М.М. ${ }^{1}$ • Стилиди И.С. • Кушлинский Н.Е.'

Актуальность. В последние 10 лет заболеваемость раком желудка значительно снизилась. Тем не менее это все еще одно из самых распространенных злокачественных новообразований в России и в мире, и проблемы ранней диагностики, прогноза и индивидуализации тактики лечения по-прежнему актуальны. Большое внимание уделяется изучению молекулярно-биологических особенностей опухоли, а также созданию на их основе многофакторных систем прогноза рака желудка. Среди потенциальных биологических маркеров опухолей важное место занимают матриксные металлопротеиназы (ММП), вовлеченные во все этапы опухолевого процесса, в первую очередь в регуляцию инвазии и метастазирования. Цель - сравнительная количественная оценка содержания некоторых представителей семейства ММП (ММП-2, 7 и 9) и одного из их тканевых ингибиторов (ТИМП-2) в опухолях и окружающей гистологически неизмененной ткани больных раком желудка; анализ взаимосвязи этих показателей с основными клинико-морфологическими особенностями и прогнозом заболевания. Материал и методы. В исследование включены 66 первичных больных раком желудка (32 мужчины и 34 женщины) в возрасте от 24 до 82 лет (медиана 61 год). Стадия I диагностирована у 22 , II - у 11 , III - у 28 , IV - у 5 пациентов. Содержание исследуемых белков определяли в экстрактах опухолей и участков гистологически неизмененной слизистой оболочки желудка с помощью стандартных наборов для прямого иммуноферментного анализа (Quantikine ${ }^{\oplus}$,
R\&D Systems, США). Результаты и обсуждение. Содержание ММП-2, 7 и 9 в опухолях статистически значимо повышено по сравнению с окружающей гистологически неизмененной слизистой оболочкой у 80,70 и $72 \%$ больных раком желудка, а увеличение уровня ТИМП-2, наблюдаемое в $61 \%$ опухолей, не достигает уровня статистической значимости. Содержание ММП-2 и ТИМП-2 в опухоли статистически значимо возрастает с увеличением индекса Т - размера и распространенности первичной опухоли ( $p<0,01$ и $p<0,05$ соответственно). Уровень ММП-2 в опухолевой ткани также возрастает с увеличением индекса $\mathrm{N}$ (вовлеченности регионарных лимфатических узлов; $p<0,01)$ и выше у больных с отдаленными метастазами, чем у пациентов без метастазов $(p<0,05)$. Содержание ММП-9 и ММП-7 в ткани рака желудка не зависит от показателей распространенности процесса. Пациенты прослежены на протяжении от 1 до 85 месяцев (медиана 18,3 месяца). По данным однофакторного анализа, высокое (более 32,6 нг/мг белка) содержание МмП-2 и низкое (менее 1,1 нг/мг белка) содержание ММП-7 в ткани рака желудка - статистически значимые факторы неблагоприятного прогноза общей выживаемости. Повышенный уровень ТИМП-2 незначительно ухудшает прогноз общей выживаемости ( $p>0,05)$, а уровень ММП-9 на него не влияет. По данным многофакторного регрессионного анализа, независимыми факторами прогноза оказались только индекс $\mathrm{T}(\mathrm{p}=0,0034)$ и уровень ММП-7 в опухолевой ткани $(\mathrm{p}=0,026)$. Заключение. у большинства обследованных больных раком желудка выявлено статистически значимое увеличение уровня экспрессии трех представителей семейства ММП - желатиназ (ММП-2 и ММП-9) и матрилизина (ММП-7) в опухолях по сравнению с окружающей гистологически неизмененной тканью. При этом только уровень ММП-2 связан с распространенностью процесса, возрастая с увеличением индексов системы TNM. Высокое содержание ММП-2 и низкое - ММП-7 в ткани рака желудка являются статистически значимыми факторами неблагоприятного прогноза общей выживаемости пациентов по данным однофакторного анализа, но только уровень ММП-7 сохраняет независимое прогностическое значение по данным многофакторного анализа.

Ключевые слова: матриксная металлопротеиназа 2, матриксная металлопротеиназа 7, матриксная металлопротеиназа 9, тканевый ингибитор матриксных металлопротеиназ 2-го типа, рак желудка, ELISA

Для цитирования: Герштейн $E C$, Иванников $A A$, Чанг ВЛ, Огнерубов НА, Давыдов ММ, Стилиди ИС, Кушлинский НЕ. Иммуноферментное исследование матриксных металлопротеиназ 2, 7, 9 и их тканевого ингибитора 2-го типа в опухолях больных раком желудка: клинико-морфологические корреляции. Альманах клинической медицины. 2018;46(4):323-9. doi: 10.18786/2072-0505-2018-464-323-329.

Поступила 22.03.2018;

принята к публикации 22.05.2018 
$\mathrm{P}$ ак желудка, несмотря на значительное снижение заболеваемости в последние 10 лет, остается одним из самых распространенных злокачественных новообразований в России и в мире, поэтому проблемы ранней диагностики, прогноза и индивидуализации тактики лечения этого тяжелого заболевания не теряют актуальности. Поскольку возможности совершенствования тактики и объема хирургического вмешательства не беспредельны, а эффективность классической химиотерапии при раке желудка весьма ограничена, надежды на дальнейший прогресс в улучшении лечения этого заболевания связаны не только с более эффективным использованием комбинированных и комплексных методов лечения, но и с разработкой принципиально новых патогенетических методов терапии, основанных на современных достижениях в изучении биохимии и молекулярной биологии опухолей.

Все больше внимания уделяется исследованию биологических свойств опухоли и созданию многофакторных систем прогноза при раке желудка [1], так как «классические» морфологические критерии прогноза не могут полностью объяснить все разнообразие биологических свойств опухоли и не всегда достаточно правильно предсказывают ее течение.

Одним из фундаментальных свойств злокачественных опухолей является способность к инвазии в окружающие ткани и метастазированию в отдаленные органы. Главное место в этих процессах отводится разрушению окружающей базальной мембраны и внеклеточного матрикса ассоциированными с опухолью протеазами. В связи с этим матриксные металлопротеиназы (ММП) - мультигенное семейство, состоящее из более 20 секретируемых и связанных с поверхностью клетки цинкзависимых эндопептидаз, участвующих в деградации практически всех компонентов внеклеточного матрикса, - уже на протяжении многих лет рассматриваются и изучаются в качестве потенциальных биологических маркеров различных опухолей [2]. К важнейшим представителям ММП относят различные коллагеназы (ММП-1, ММП-8 и др.), желатиназы А и В (ММП-2 и ММП-9 соответственно), матрилизин (ММП-7), стромелизины (ММП-3 и ММП-10). Помимо большинства компонентов внеклеточного матрикса субстратами ММП могут быть другие протеазы, хемотаксические молекулы, латентные формы факторов роста, растворимые и мембранно-ассоциированные белки, связывающие факторы роста.
Герштейн Елена Сергеевна - д-р биол. наук, профессор, вед. науч. сотр. лаборатории клинической биохимии $\triangle 115478$, Каширское шоссе, 24, Российская Федерация.

Тел.: +7 (499) 3241159. E-mail: biochimia @yandex.ru

Иванников Андрей

Андреевич - хирургонколог, ассистент кафедры анатомии, оперативной хирургии и онкологии ${ }^{2}$

Чанг Виктор Луисович ассистент кафедры анатомии, оперативной хирургии и онкологии ${ }^{2}$

Огнерубов Николай Алексеевич - д-р мед. наук, профессор, зав. кафедрой анатомии, оперативной хирургии и онкологии ${ }^{2}$

Давыдов Михаил Михайлович - д-р мед. наук, чл.-корр. РАН, зав. отделением торакальной онкологии'

\section{Стилиди Иван}

Сократович - д-р мед. наук, профессор, чл.-корр. РАН, зав. хирургическим отделением абдоминальной онкологии, директор' Кушлинский Николай Евгеньевич - д-р мед. наук, профессор, чл.-корр. РАН, зав. лабораторией клинической биохимии'

1 ФГБУ «Национальный медицинский исследовательский центр онкологии им. Н.Н. Блохина» Минздрава России; 115478 , г. Москва, Каширское шоссе, 24 Российская Федерация 2 ФГБОУ ВО «Тамбовский государственный университет им. Г.Р. Державина»; 392000, г. Тамбов, ул. Интернациональная, 33, Российская Федерация
ММП вовлечены во все этапы опухолевого процесса, но особенно велико их значение в регуляции инвазии и метастазирования. Они участвуют и в регуляции опухолевого ангиогенеза как посредством комплексного взаимодействия c VEGF и его рецепторами, так и независимо от этой ключевой проангиогенной системы [3].

Активность ММП в межклеточном пространстве специфически подавляется тканевыми ингибиторами (ТИМП) - структурно родственными белками, три из которых (ТИМП-1, 2 и 4) секретируются в растворимой форме, а один (ТИМП-3) связан с внеклеточным матриксом [4]. ТИМП, в первую очередь ТИМП-1 и 2, играют важную самостоятельную роль в регуляции роста и дифференцировки опухолевых и нормальных клеток, в том числе участвуя в регуляции ангиогенеза [5].

Увеличение экспрессии ММП обнаружено в опухолях различного генеза, при этом активация происходит по паракринному механизму с участием факторов роста и цитокинов, секретируемых инфильтрирующими опухоль макрофагами и лимфоцитами, а также клетками опухолевой стромы [6]. Тканевая экспрессия, а в некоторых случаях и сывороточные уровни отдельных ММП и ТИМП, имеют прогностическое и/или диагностическое значение при таких заболеваниях, как рак яичников, колоректальный рак, рак почки и др. [2]. В последние годы появились обобщающие публикации о роли тканевой экспрессии некоторых ММП при раке желудка [7-11]. В большинстве таких исследований либо проводили иммуногистохимическое окрашивание соответствующих белков, либо оценивали экспрессию мРНК методами полимеразной цепной реакции.

Цель данного исследования - сравнительная количественная оценка содержания некоторых представителей семейства ММП (ММП-2, 7 и 9) и одного из их тканевых ингибиторов (ТИМП-2) в опухолях и окружающей гистологически неизмененной ткани больных раком желудка, анализ взаимосвязи этих показателей с основными клинико-морфологическими особенностями и прогнозом заболевания.

\section{Материал и методы}

В исследование включены 66 первичных больных раком желудка (32 мужчины и 34 женщины) в возрасте от 24 до 82 лет (медиана 61 год), проходивших обследование и лечение в ФГБУ «РОНЦ им. Н.Н. Блохина» РАМН в 2010-2011 гг. Диагноз рака желудка у всех включенных в исследование пациентов подтвержден данными планового 
гистологического исследования. Дизайн работы соответствует требованиям этического комитета ФГБУ «НМИЦ онкологии им. Н.Н. Блохина» Минздрава России.

Стадия I диагностирована у 22, II - у 11, III у $28, \mathrm{IV}$ - у 5 пациентов. У $27 \%$ пациентов опухоль локализовалась в теле, у 21\% - в проксимальном, у $22 \%$ - в антральном отделе желудка. Кроме того, у 13\% больных были одновременно поражены тело и проксимальный отдел желудка, еще у $13 \%$ - тело и антральный отдел. Тотальное поражение было констатировано у 4\% больных.

Образцы опухолевой и гистологически неизмененной ткани желудка (200-300 мг) брали во время операции и немедленно замораживали для дальнейшего хранения при $-70{ }^{\circ} \mathrm{C}$.

Содержание исследуемых белков определяли в экстрактах опухолей и участков гистологически неизмененной слизистой оболочки желудка с помощью стандартных наборов для прямого иммуноферментного анализа: Human/Mouse/ Rat MMP-2 (total), Human MMP-7 (total), Human MMP-9 (total) и Human TIMP-2 (Quantikine ${ }^{\varpi}, \mathrm{R} \& \mathrm{D}$ Systems, CША) в соответствии с инструкциями производителя. Для проведения иммуноферментного анализа опухолевых и неизмененных тканей желудка получали их экстракты как описано ранее [12]. Измерения проводили на автоматическом универсальном ридере для микропланшета $\mathrm{EL}_{\mathrm{X}} 800$ (Bio-Tek Instruments, Inc., США). Концентрацию маркеров выражали в нанограммах на 1 мг общего белка (нг/мг белка), определенного в экстрактах тканей по методу Лоури.

При сравнении и анализе взаимосвязи показателей использовали непараметрические критерии Манна - Уитни, Краскела - Уоллиса, Вилкоксона, тест корреляции рангов Спирмена (R). Выживаемость больных оценивали методом Каплана - Мейера, а также с помощью регрессионной многофакторной модели Кокса. Данные обрабатывали с помощью программы Statistica 6.0 (StatSoft Inc).

\section{Результаты и обсуждение}

Содержание всех исследованных ММП было статистически значимо повышено по сравнению с гистологически неизмененной тканью в опухолях 80 (ММП-2), 70 (ММП-7) и 72\% (ММП-9) больных раком желудка (табл. 1). У 61\% больных отмечено также увеличение содержания ТИМП-2, однако медианы этого показателя в опухоли и неизмененной слизистой желудка (18,8 и 16,7 нг/мг белка), в отличие от исследованных ММП, значимо не различались (см. табл. 1).
Таблица 1. Содержание (нг/мг белка) ММП-2, 7, 9 и ТИМП-2 в опухолях и гистологически неизмененной слизистой оболочке больных раком желудка

\begin{tabular}{|c|c|c|c|c|c|}
\hline \multirow[t]{2}{*}{ Показатель } & \multicolumn{2}{|c|}{ Опухоль (Т) } & \multicolumn{2}{|c|}{ Слизистая оболочка (N) } & \multirow{2}{*}{$\mathrm{T}>\mathrm{N}, \%$} \\
\hline & медиана & диапазон & медиана & диапазон & \\
\hline ММП-2 & $32,6^{*}$ & $4,4-127$ & 15,9 & $4-249$ & 80 \\
\hline ММП-7 & $1,1^{*}$ & $0-16,6$ & 0 & $0-4,1$ & 70 \\
\hline ММП-9 & $150^{+}$ & $0-2000$ & 77,9 & $0-563$ & 72 \\
\hline ТИМП-2 & 18,8 & $0-100$ & 16,7 & $5,4-56,3$ & 61 \\
\hline
\end{tabular}

ММП - матриксная металлопротеиназа, ТИМП - тканевый ингибитор матриксной металлопротеиназы

"p $<0,0001,{ }^{\dagger} p<0,01$ по сравнению с неизмененной слизистой (парный критерий Вилкоксона)

Слабая, но статистически значимая положительная корреляционная связь показателей опухолевой и гистологически неизмененной ткани выявлена для трех маркеров: ММП-7 ( $\mathrm{R}=039$; $\mathrm{p}=0,0012), \quad$ ММП-9 $\quad(\mathrm{R}=0,38 ; \quad \mathrm{p}=0,0024)$ и ТИМП-2 $(\mathrm{R}=0,35 ; \mathrm{p}=0,028)$. Кроме того, уровни ММП-2 и ТИМП-2 положительно коррелировали между собой как в опухолевой $(\mathrm{R}=0,58 ; \mathrm{p}<0,0001)$, так и в неизмененной слизистой желудка $(\mathrm{R}=0,59$; $\mathrm{p}<0,0001)$

Для того чтобы оценить клиническое значение определения исследуемых маркеров в ткани рака желудка, мы проанализировали взаимосвязь их уровней с основными клинико-морфологическими особенностями заболевания: локализацией, типом роста и гистологическим строением первичной опухоли, ее размером и распространенностью (Т), степенью поражения регионарных лимфатических узлов $(\mathrm{N})$, наличием отдаленных метастазов (M). Значимых ассоциаций с гистологическим строением, степенью дифференцировки, локализацией, типом роста первичной опухоли не выявлено.

В табл. 2 даны уровни маркеров в зависимости от индексов системы TNM. Отмечено статистически значимое увеличение уровней ММП-2 и ТИМП-2 с увеличением индекса Т - размера и распространенности первичной опухоли ( $\mathrm{p}<0,01$ и $\mathrm{p}<0,05$ соответственно; тест Краскела Уоллиса). Уровень ММП-2 в опухолевой ткани также значимо возрастал с увеличением индекса $\mathrm{N}$ (вовлеченность регионарных лимфатических узлов; $\mathrm{p}<0,01)$ и был выше у больных с отдаленными метастазами, чем у пациентов без метастазов $(\mathrm{p}<0,05)$. Содержание ММП-9 и ММП-7 в ткани рака желудка не зависело от показателей распространенности процесса.

Вошедшие в настоящее исследование 63 пациента из 66 были прослежены на протяжении 
Таблица 2. Содержание (нг/мг белка) ММП-2, 7, 9 и ТИМП-2 в опухолях больных раком желудка с учетом показателей распространенности процесса по системе TNM

\begin{tabular}{|c|c|c|c|c|c|c|c|c|c|}
\hline \multirow[t]{2}{*}{ Индекс } & \multirow[t]{2}{*}{$\mathrm{N}$} & \multicolumn{2}{|l|}{ ММП-2 } & \multicolumn{2}{|l|}{ ММП-7 } & \multicolumn{2}{|l|}{ ММП-9 } & \multicolumn{2}{|l|}{ тимп-2 } \\
\hline & & медиана & диапазон & медиана & диапазон & медиана & диапазон & медиана & диапазон \\
\hline \multicolumn{10}{|c|}{ Размер и распространенность первичной опухоли (Т) } \\
\hline $\mathrm{T}_{1}$ & 13 & 13,9 & $6,2-103$ & 0,4 & $0-11,3$ & 131 & $0-343$ & 11,5 & $0-28,7$ \\
\hline $\mathrm{T}_{2}$ & 5 & 32,6 & $15,7-44,8$ & 1,1 & $0-3,1$ & 170 & $19,5-256$ & 21,1 & $16,8-35,9$ \\
\hline $\mathrm{T}_{3}$ & 27 & 38,1 & $8,8-96,2$ & 1,7 & $0-16,6$ & 156 & $2,5-2000$ & 17,8 & $9,5-100$ \\
\hline $\mathrm{T}_{4}$ & 14 & 43,0 & $14,5-123^{*}$ & 1,4 & $0-10,4$ & 256 & $25,9-573$ & 25,3 & $15,4-45,2^{\dagger}$ \\
\hline \multicolumn{10}{|c|}{ Метастазы в лимфатических узлах (N) } \\
\hline $\mathrm{N}_{0}$ & 24 & 21,9 & $6,2-103$ & 1 & $0-11,4$ & 138 & $0-573$ & 15,2 & $0-44,1$ \\
\hline $\mathrm{N}_{1}$ & 26 & 34,7 & $12-123$ & 1,1 & $0-10,4$ & 156 & $19,5-2000$ & 19 & $9,5-100$ \\
\hline $\mathrm{N}_{2}$ & 9 & 45,2 & $8,8-96,2^{\ddagger}$ & 2,8 & $0-16,6$ & 340 & $2,5-447$ & 24,7 & $15-41,4$ \\
\hline \multicolumn{10}{|c|}{ Отдаленные метастазы (М) } \\
\hline$M_{0}$ & 48 & 30,5 & $6,2-103$ & 1,4 & $0-16,6$ & 148 & $0-2000$ & 17,5 & $0-100$ \\
\hline $\mathrm{M}_{1}$ & 11 & 49,3 & $15,2-123^{\S}$ & 0,5 & $0-10,4$ & 262 & $25,9-485$ & 23 & $19,1-45,2$ \\
\hline
\end{tabular}

ММП - матриксная металлопротеиназа, ТИМП - тканевый ингибитор матриксной металлопротеиназы

${ }^{*} \mathrm{p}\left(\mathrm{T}_{4}-\mathrm{T}_{0}\right)<0,01$ (тест Краскела - Уоллиса)

${ }^{\dagger} \mathrm{p}\left(\mathrm{T}_{4}-\mathrm{T}_{0}\right)<0,05$ (тест Краскела - Уоллиса)

${ }^{\ddagger} \mathrm{p}\left(\mathrm{N}_{2}-\mathrm{N}_{0}\right)<0,01$ (тест Краскела - Уоллиса)

${ }^{\S} \mathrm{p}\left(\mathrm{M}_{1}-\mathrm{M}_{0}\right)<0,05$ (тест Манна - Уитни)

от 1 до 85 месяцев (медиана 18,3 месяца). За этот период 33 из них (53\%) умерли от прогрессирования основного заболевания в сроки от 1 до 33 месяцев (медиана 7,6 месяца). В качестве пограничных значений для оценки общей выживаемости были приняты показатели медианных уровней исследованных маркеров в ткани рака желудка: 32,6 нг/мг белка - для ММП-2; 1,1 нг/мг белка - для ММП-7; 150 нг/мг белка для ММП-9 и 18,8 нг/мг белка - для ТИМП-2.

Фактором неблагоприятного прогноза общей выживаемости больных раком желудка оказался высокий уровень ММП-2 в опухоли (рис. 1). При содержании маркера ниже порогового уровня 5-летняя выживаемость составила 58\% (медиана не достигнута), при более высоком - 33\% (медиана выживаемости - 13 месяцев; $\mathrm{p}<0,01)$.

Выживаемость пациентов также была хуже при высоком уровне ТИМП-2 в опухоли (рис. 2), чем при низком уровне маркера (43 и $54 \%$ соответственно при 5-летнем сроке наблюдения), но это различие не достигало уровня статистической значимости $(\mathrm{p}=0,305)$.

Парадоксально, но 5-летняя выживаемость больных с высоким содержанием
ММП-7 в опухоли оказалась на $14 \%$ хуже, чем у пациентов с низким уровнем маркера (рис. 3; $\mathrm{p}<0,05)$. Медиана выживаемости в первой группе составила 19 месяцев, во второй - не была достигнута за весь период наблюдения.

Содержание ММП-9 в опухолевой ткани практически не влияло на общую выживаемость больных раком желудка: 5-летняя выживаемость пациентов с уровнем маркера в опухоли более 150 нг/мг белка составила 46\%, пациентов с содержанием маркера ниже этого уровня - 50\%; медианы 28 и 33 месяца соответственно.

Таким образом, по данным однофакторного анализа, статистически значимыми факторами неблагоприятного прогноза общей выживаемости оказались лишь высокое содержание ММП-2 и низкое - ММП-7 в ткани рака желудка. Важно отметить: уровень ММП-2 статистически значимо положительно ассоциирован с показателями системы TNM, характеризующими распространенность процесса, что может отражаться на его прогностическом значении. Действительно, по данным многофакторного регрессионного анализа, независимыми факторами прогноза были только индекс $\mathrm{T}(\mathrm{p}=0,0034)$ и уровень ММП-7 в опухолевой ткани $(\mathrm{p}=0,026)$. 


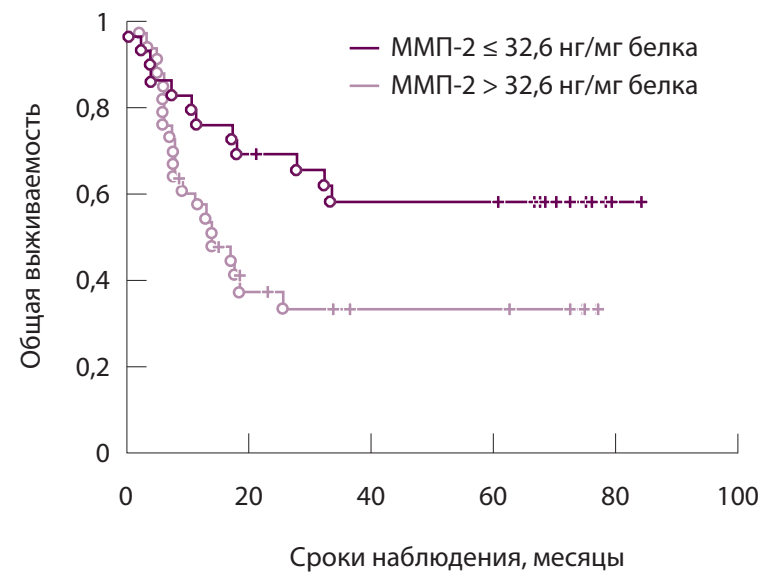

Рис. 1. Общая выживаемость больных раком желудка в зависимости от содержания матриксной металлопротеиназы (ММП)-2 в опухоли $(\mathrm{p}<0,01)$

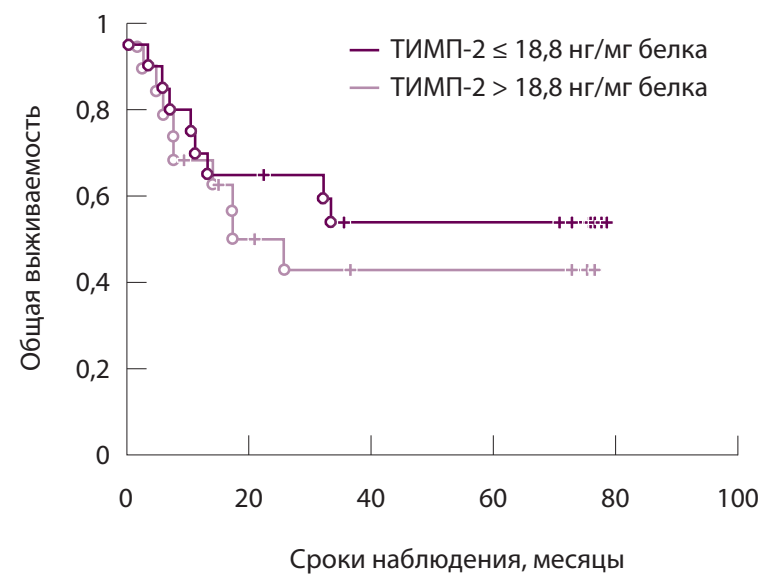

Рис. 2. Общая выживаемость больных раком желудка в зависимости от содержания тканевого ингибитора матриксной металлопротеиназы (ТИМП)-2 в опухоли $(p=0,035)$

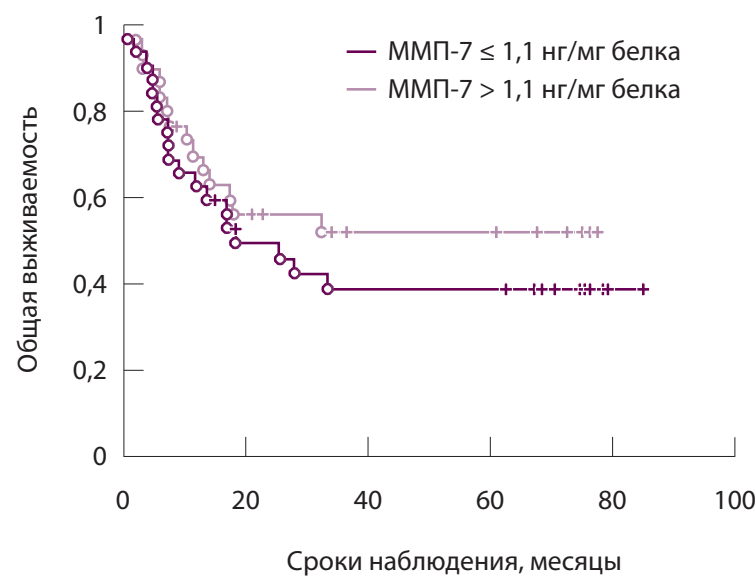

Рис. 3. Общая выживаемость больных раком желудка в зависимости от содержания матриксной металлопротеиназы (ММП)-7 в опухоли $(\mathrm{p}<0,05)$

\section{Конфликт интересов}

Авторы статьи заявляют об отсутствии конфликтов интересов.

Финансирование

Работа проведена без привлечения дополнительного финансирования со стороны третьих лиц.
Опубликованы данные о том, что гиперэкспрессия некоторых ММП, в том числе исследованных нами ММП-2, 7 и 9, является фактором неблагоприятного прогноза выживаемости больных раком желудка $[9-11,13,14]$. Так, по результатам метаанализа данных 10 независимых исследований, повышенная экспрессия ММП-2 увеличивает риск смерти от рака желудка в 1,92 раза (95\% доверительный интервал 1,48-2,48) [9]. Обращает на себя внимание, что все работы, вошедшие в этот анализ, выполнены полуколичественным иммуногистохимическим методом, и прогностическое значение ММП-2 было выявлено только в 6 из них. Иммуногистохимическим методом выполнены 6 из 7 работ, вошедших в метаанализ прогностического значения ММП-7 [10], а также 9 из 11 проанализированных исследований тканевой экспрессии ММП-9 [11]. Соответственно, прямое сравнение наших количественных данных с результатами этих публикаций не вполне корректно, хотя нужно подчеркнуть, что нам также удалось продемонстрировать неблагоприятное прогностическое значение высокого содержания ММП-2 в ткани рака желудка. Вместе с тем две группы исследователей, использовавшие иммуноферментный метод оценки экспрессии ММП-9 [15, 16], не нашли прогностического значения для этого маркера, что также соответствует нашим результатам.

\section{Заключение}

у большинства обследованных больных раком желудка выявлено статистически значимое увеличение уровня экспрессии трех представителей семейства ММП - желатиназ (ММП-2 и ММП-9) и матрилизина (ММП-7) - в опухолях по сравнению с окружающей гистологически неизмененной тканью. При этом только уровень ММП-2 был связан с распространенностью процесса, возрастая с увеличением индексов системы TNM. Уровень ТИМП-2 был повышен в опухолях $61 \%$ пациентов (различия не достигли уровня статистической значимости) и статистически значимо возрастал с увеличением индекса Т.

Высокое содержание ММП-2 и низкое ММП-7 в ткани рака желудка являются статистически значимыми факторами неблагоприятного прогноза общей выживаемости пациентов по данным однофакторного анализа, но только уровень ММП-7 сохраняет независимое прогностическое значение по данным многофакторного анализа. Повышенный уровень ТИМП-2 незначительно ухудшает прогноз общей выживаемости, а уровень ММП-9 на него не влияет. 


\section{Литература}

1. Wu W, Yong WW, Chung MC. A simple biomarker scoring matrix for early gastric cancer detection. Proteomics. 2016;16(22):2921-30. doi: 10.1002/pmic.201600194.

2. Герштейн ЕС, Кушлинский НЕ. Клинические перспективы исследования ассоциированных с опухолью протеаз и их тканевых ингибиторов у онкологических больных. Вестник Российской академии медицинских наук. 2013;68(5):16-27. doi: 10.15690/vramn. v68i5.659.

3. Deryugina El, Quigley JP. Pleiotropic roles of matrix metalloproteinases in tumor angiogenesis: contrasting, overlapping and compensatory functions. Biochim Biophys Acta. 2010;1803(1):103-20. doi: 10.1016/j.bbamcr.2009.09.017.

4. Ramnath N, Creaven PJ. Matrix metalloproteinase inhibitors. Curr Oncol Rep. 2004;6(2): 96-102. doi: 10.1007/s11912-004-0020-7.

5. Sounni NE, Janssen M, Foidart JM, Noel A. Membrane type-1 matrix metalloproteinase and TIMP-2 in tumor angiogenesis. Matrix Biol. 2003;22(1):55-61. doi: 10.1016/S0945053X(03)00003-9.

6. Westermarck J, Kähäri VM. Regulation of matrix metalloproteinase expression in tumor invasion. FASEB J. 1999;13(8):781-92. doi: 10.1096/fasebj.13.8.781.

\section{References}

1. Wu W, Yong WW, Chung MC. A simple biomarker scoring matrix for early gastric cancer detection. Proteomics. 2016;16(22):2921-30. doi: 10.1002/pmic.201600194.

2. Gerstein ES, Kushlinskii NE. Clinical prospects of tumor-associated proteases and their tissue inhibitors investigation in oncologic patients. Annals of the Russian Academy of Medical Sciences. 2013;68(5):16-27. Russian. doi: 10.15690/vramn.v68i5.659.

3. Deryugina El, Quigley JP. Pleiotropic roles of matrix metalloproteinases in tumor angiogenesis: contrasting, overlapping and compensatory functions. Biochim Biophys Acta. 2010;1803(1):103-20. doi: 10.1016/j.bbamcr.2009.09.017.

4. Ramnath N, Creaven PJ. Matrix metalloproteinase inhibitors. Curr Oncol Rep. 2004;6(2): 96-102. doi: 10.1007/s11912-004-0020-7.

5. Sounni NE, Janssen M, Foidart JM, Noel A. Membrane type-1 matrix metalloproteinase and TIMP-2 in tumor angiogenesis. Matrix Biol. 2003;22(1):55-61. doi: 10.1016/S0945053X(03)00003-9.

6. Westermarck J, Kähäri VM. Regulation of matrix metalloproteinase expression in tumor invasion. FASEB J. 1999;13(8):781-92. doi: 10.1096/fasebj.13.8.781.
7. Chen J, Chen LJ, Zhou HC, Yang RB, Lu Y, Xia YL, Wu W, Hu LW. Prognostic value of matrix metalloproteinase-9 in gastric cancer: a meta-analysis. Hepatogastroenterology. 2014;61(130): 518-24.

8. Sampieri CL, León-Córdoba K, Remes-Troche JM. Matrix metalloproteinases and their tissue inhibitors in gastric cancer as molecular markers. J Cancer Res Ther. 2013;9(3):356-63. doi: 10.4103/0973-1482.119302.

9. Shen W, Xi H, Wei B, Chen L. The prognostic role of matrix metalloproteinase 2 in gastric cancer: a systematic review with meta-analysis. J Cancer Res Clin Oncol. 2014;140(6):1003-9. doi: 10.1007/s00432-014-1630-6.

10. Soleyman-Jahi S, Nedjat S, Abdirad A, Hoorshad N, Heidari R, Zendehdel K. Prognostic significance of matrix metalloproteinase-7 in gastric cancer survival: a meta-analysis. PLoS One. 2015;10(4):e0122316. doi: 10.1371/journal.pone.0122316.

11. Zhang QW, Liu L, Chen R, Wei YQ, Li P, Shi HS, Zhao YW. Matrix metalloproteinase-9 as a prognostic factor in gastric cancer: a meta-analysis. Asian Pac J Cancer Prev. 2012;13(6): 2903-8. doi: 10.7314/APJCP.2012.13.6.2903.

12. Герштейн EC, Короткова EA, Щербаков АM, Пророков ВВ, Головков ДА, Кушлинский НЕ. Матриксные металлопротеиназы 7 и 9 и их тканевые ингибиторы 1 и 4 типа в опухо-

7.Chen J, Chen LJ, Zhou HC, Yang RB, Lu Y, Xia YL, Wu W, Hu LW. Prognostic value of matrix metalloproteinase-9 in gastric cancer: a meta-analysis. Hepatogastroenterology. 2014;61(130):518-24.

8. Sampieri CL, León-Córdoba K, Remes-Troche JM. Matrix metalloproteinases and their tissue inhibitors in gastric cancer as molecular markers. J Cancer Res Ther. 2013;9(3):356-63. doi: 10.4103/0973-1482.119302.

9. Shen W, Xi H, Wei B, Chen L. The prognostic role of matrix metalloproteinase 2 in gastric cancer: a systematic review with meta-analysis. J Cancer Res Clin Oncol. 2014;140(6):10039. doi: 10.1007/s00432-014-1630-6.

10. Soleyman-Jahi S, Nedjat S, Abdirad A, Hoorshad N, Heidari R, Zendehdel K. Prognostic significance of matrix metalloproteinase-7 in gastric cancer survival: a meta-analysis. PLoS One. 2015;10(4):e0122316. doi: 10.1371/journal.pone. 0122316 .

11.Zhang QW, Liu L, Chen R, Wei YQ, Li P, Shi HS, Zhao YW. Matrix metalloproteinase-9 as a prognostic factor in gastric cancer: a meta-analysis. Asian Pac J Cancer Prev. 2012;13(6): 2903-8. doi: 10.7314/APJCP.2012.13.6.2903.

12. Gershtein ES, Korotkova EA, Shcherbakov AM, Prorokov VV, Golovkov DA, Kushlinskii NE. Matrix metalloproteinases 7 and 9 and their types 1 and 4 tissue inhibitors in tumors and лях и плазме крови больных раком толстой кишки. Бюллетень экспериментальной биологии и медицины. 2007;143(4):438-41.

13. Kubben FJ, Sier CF, van Duijn W, Griffioen G, Hanemaaijer $\mathrm{R}$, van de Velde $\mathrm{CJ}$, van Kriek en JH, Lamers CB, Verspaget HW. Matrix metalloproteinase- 2 is a consistent prognostic factor in gastric cancer. Br J Cancer. 2006;94(7): 1035-40. doi: 10.1038/sj.bjc.6603041.

14. Mrena J, Wiksten JP, Nordling S, Kokkola A, Ristimäki A, Haglund C. MMP-2 but not MMP-9 associated with COX-2 and survival in gastric cancer. J Clin Pathol. 2006;59(6):618-23. doi: 10.1136/jcp.2005.033761.

15. de Mingo $M$, Morán $A$, Sánchez-Pernaute $A$, Iniesta P, Díez-Valladares L, Pérez-Aguirre $E$, de Juan C, García-Aranda C, Díaz-López A, García-Botella A, Martín-Antona E, Benito M, Torres A, Balibrea JL. Expression of MMP-9 and TIMP-1 as prognostic markers in gastric carcinoma. Hepatogastroenterology. 2007;54(73): 315-9.

16. Kubben FJ, Sier CF, Hawinkels LJ, Tschesche H, van Duijn W, Zuidwijk K, van der Reijden JJ, Hanemaaijer R, Griffioen G, Lamers CB, Verspaget HW. Clinical evidence for a protective role of lipocalin-2 against MMP-9 autodegradation and the impact for gastric cancer. Eur J Cancer. 2007;43(12):1869-76. doi: 10.1016/j. ejca.2007.05.013.

plasma of patients with colorectal cancer. Bull Exp Biol Med. 2007;143(4):459-62.

13. Kubben FJ, Sier CF, van Duijn W, Griffioen G, Hanemaaijer $R$, van de Velde $C J$, van Krieken JH, Lamers $\mathrm{CB}$, Verspaget HW. Matrix metalloproteinase- 2 is a consistent prognostic factor in gastric cancer. Br J Cancer. 2006;94(7): 1035-40. doi: 10.1038/sj.bjc.6603041.

14. Mrena J, Wiksten JP, Nordling S, Kokkola A, Ristimäki A, Haglund C. MMP-2 but not MMP-9 associated with COX-2 and survival in gastric cancer. J Clin Pathol. 2006;59(6):61823. doi: $10.1136 /$ jcp. 2005.033761.

15. de Mingo M, Morán A, Sánchez-Pernaute $A$, Iniesta $P$, Díez-Valladares $L$, Pérez-Aguirre $E$, de Juan C, García-Aranda C, Díaz-López A, García-Botella A, Martín-Antona E, Benito M, Torres A, Balibrea JL. Expression of MMP-9 and TIMP-1 as prognostic markers in gastric carcinoma. Hepatogastroenterology. 2007;54(73): 315-9.

16. Kubben FJ, Sier CF, Hawinkels LJ, Tschesche H, van Duijn W, Zuidwijk K, van der Reijden JJ, Hanemaaijer R, Griffioen G, Lamers CB, Verspaget HW. Clinical evidence for a protective role of lipocalin-2 against MMP-9 autodegradation and the impact for gastric cancer. Eur J Cancer. 2007;43(12):1869-76. doi: 10.1016/j. ejca.2007.05.013. 


\title{
ELISA study of matrix metalloproteinases 2, 7, 9 and their type 2 tissue inhibitor in the tumors of gastric cancer patients: clinical and pathologic correlations
}

\author{
E.S. Gershtein • A.A. Ivannikov² • V.L. Chang² • N.A. Ognerubov² \\ M.M. Davydov ${ }^{1} \cdot$ I.S. Stilidi ${ }^{1} \cdot$ N.E. Kushlinskii ${ }^{1}$
}

Background: Over the last 10 years the incidence of gastric cancer has declined significantly. Nevertheless, it remains one of the most prevalent malignancies both in Russia and worldwide. Therefore, the problems of early diagnostics, prognosis and individualized treatment choice are still on the agenda. Much attention is paid to the evaluation of molecular biological characteristics of the tumor, as well as to the development of multiparametric prognostic systems for gastric cancer based on its identified characteristics. An important place among potential tumor biological markers belongs to matrix metalloproteinases (MMPs) involved into all the stages of tumor progression, first of all, into the regulation of invasion and metastasizing. Aim: Comparative quantitative evaluation of some MMP family members (MMP-2, 7, and 9) and one of the tissue MMP inhibitors (TIMP-2) levels in the tumors and adjacent histologically unchanged mucosa in gastric cancer patients, the analysis of their associations with the main clinical and pathological features of the disease and its prognosis. Materials and methods: Sixty six (66) primary gastric cancer patients ( 32 male and $34 \mathrm{fe}$ male) aged 24 to 82 years (median, 61 year) were recruited into the study. Twenty two (22) patients were with stage I of the disease, 11 with stage II, 28 with stage III, and 5 with stage IV. The concentrations of the proteins studied were measured in the tumor and unchanged mucosa extracts by standard direct ELISA kits (Quantikine ${ }^{\oplus}$, R\&D Systems, USA). Results: Tumor MMP-2, 7 and 9 levels were significantly increased, compared to those in the adjacent histologically unchanged mucosa, in 80 , 70 and $72 \%$ of gastric cancer patients, respectively, while the increase of TIMP-2 level found in $61 \%$ of the tumors was not statistically significant. Tumor MMP-2 and TIMP-2 content was increasing significantly with higher $T$ index - size and advancement of the primary tumor $(p<0.01$ and $p<0.05$ respectively). Tumor MMP-2 level was also increasing in parallel with the $\mathrm{N}$ index (regional lymph node involvement; $p<0.01$ ); it was significantly higher in the patients with distant metastases than in those without them $(p<0.05)$. Tumor MMP-9 and MMP-7 concentrations were not significantly associated with the indices of the tumor progression The patients were followed up for 1 to 85 months (median, 18.3 months). According to the univariate analysis, high (>32.6 ng/mg protein) MMP-2 and low MMP-7 $(<1.1 \mathrm{ng} / \mathrm{mg}$ protein) levels in the gastric cancer tissue represent statistically significant unfavorable prognostic factors for overall survival. Increased TIMP-2 level is associated with a non-significant decrease in the overall survival $(p>0.05)$, whereas the MMP-9 level was unrelated to the gastric cancer prognosis. Only $T$ index $(p=0.0034)$ and tumor MMP-7 content $(p=0.026)$ remained independent prognostic factors in the multivariate regression analysis. Conclusion: The majority of gastric cancer patients demonstrate a significant increase in the expression of three MMP family members, i.e. gelatinases (MMP-2 and 9), and matrilysin (MMP-7), in the tumors, as compared to adjacent histologically unchanged mucosa. Only MMP-2 levels were associated with the disease progression, increasing with higher TNM system indices. High MMP-2 and low MMP-7 content in the gastric cancer tissue are significant unfavorable prognostic factors for the overall survival in the univariate analysis, but only MMP-7 has retained its independent prognostic value in the multivariate assessment.

Key words: matrix metalloproteinase 2, matrix metalloproteinase 7, matrix metalloproteinase 9, tissue inhibitor of matrix metalloproteinases 2, gastric cancer, ELISA

For citation: Gershtein ES, Ivannikov AA, Chang VL, Ognerubov NA, Davydov MM, Stilidi IS, Kushlinskii NE. ELISA study of matrix metalloproteinases 2, 7,9 and their type 2 tissue inhibitor in the tumors of gastric cancer patients: clinical and pathologic correlations. Almanac of Clinical Medicine. 2018;46(4):323-9. doi: 10.18786/2072-0505-2018-46-4-323-329.

Received 22 March 2018; accepted 22 May 2018
Elena S. Gershtein - ScD in Biology, Professor, Leading Research Fellow, Laboratory of Clinical Biochemistry

$\triangle 24$ Kashirskoe shosse, Moscow, 115478, Russian Federation. Tel.: +7 (499) 3241159

E-mail: biochimia@yandex.ru

Andrey A. Ivannikov - Surgical Oncologist, Assistant of the Chair of Anatomy, Operative Surgery and Oncology

Victor L. Chang - Assistant of the Chair of Anatomy, Operative Surgery and Oncology ${ }^{2}$

Nikolay A. Ognerubov - MD, PhD, Professor, Head of the Chair of Anatomy, Operative Surgery and Oncology ${ }^{2}$

Mikhail M. Davydov - MD, PhD, MemberCorrespondent of Russian Academy of Sciences, Head of the Department of Thoracic Oncology ${ }^{1}$

Ivan S. Stilidi - MD, PhD, Professor, MemberCorrespondent of Russian Academy of Sciences, Head of the Surgical Department of Abdominal Oncology, Director

Nikolay E. Kushlinskii - MD, PhD, Professor, Member-Correspondent of Russian Academy of Sciences, Head of Laboratory of Clinical Biochemistry
N.N. Blokhin National Medical Research Centre of Oncology; 24 Kashirskoe shosse, Moscow, 115478, Russian Federation

2 Tambov State University named after G.R. Derzhavin; 33 Internatsional'naya ul., Tambov, 392000, Russian Federation 